\title{
Preserving the liver for transplantation
}

\section{c6 \\ Liver transplan- \\ tation ... \\ is severely hampered by a shortage of donor livers}

Normothermic machine perfusion (NMP), in which a donor liver is maintained in a physiological state (at $37^{\circ} \mathrm{C}$ and perfused with oxygenated blood containing nutrients and medications), improved graft preservation time, organ utilization and decreased graft injury after transplantation compared with cold storage, reports a trial published in Nature. The trial demonstrates that NMP is feasible and effective for organ preservation and, if successfully translated to clinical practice, could reduce tissue injury associated with liver transplants and enable better assessment of organ quality to ultimately improve liver transplantation outcomes.

Liver transplantation is a successful treatment for end-stage liver disease, but this approach is severely hampered by a shortage of donor livers.

"Approximately one in five patients died on the UK liver transplant waiting list last year whilst, paradoxically, almost 500 livers from deceased organ donors were not retrieved or not transplanted," explains author Peter Friend. "This was because the suboptimal condition of many donor organs is such that these are unlikely to work after being preserved in an ice box - despite many advances in liver transplantation, the method of organ preservation has scarcely changed in 30 years."

David Nasralla and colleagues sought to directly compare the

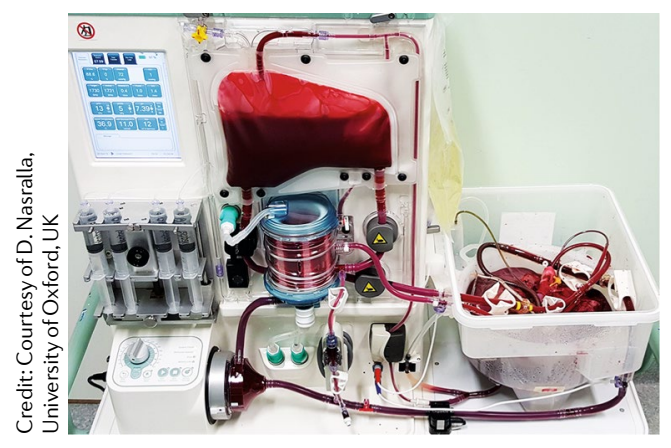

traditional method of static cold storage (SCS) of the liver graft with that of NMP. In a multinational, randomized, controlled trial, livers from adult donors who had been declared dead by cardiovascular criteria (DCD) or brainstem death (DBD) were eligible for enrolment to be potentially matched with adults awaiting a liver-only transplant across seven liver transplantation centres in the UK, Belgium, Spain and Germany. If a suitable liver was allocated to a recipient then the liver graft was randomly assigned $(n=335$ total; 64 livers excluded) to either SCS or NMP after removal from the donor. Biochemical data and graft and patient survival were recorded daily during the first postoperative week and at 10 days, 30 days, 6 months and 12 months. At 6 months, biliary injury was assessed by magnetic resonance cholangiopancreatography and the primary endpoint was defined as the difference in peak levels of serum aspartate aminotransferase (AST) within 7 days after transplantation, a clinically accepted biomarker of long-term graft survival.

101 SCS and 121 NMP livers were successfully transplanted, with higher discard rates for SCS $(24.1 \%, 32$ of 133) than for NMP (11.7\%, 16 of 137). Total preservation time, as measured from the start of cold aortic perfusion in the donor until graft perfusion in the recipient, was longer for NMP than SCS livers (11 h $54 \mathrm{~min}$ versus $7 \mathrm{~h} 45 \mathrm{~min}$; $P<0.001)$. No statistically significant difference was observed in preservation time between DBD and DCD donor livers. Notably, perfusion characteristics indicative of organ quality could be closely monitored during NMP.

Compared with SCS, peak AST levels during the first 7 days after transplantation were reduced by $49.4 \%$ with NMP when adjusted by centre and donor type $(P<0.001)$.
Interestingly, the effect of NMP was different in the two donor types with the reduction in geometric mean peak AST level greater in DCD than in DBD livers (73.3\% versus $40.2 \%$ ). Moreover, the odds of developing early allograft dysfunction were $74 \%$ lower in the NMP group (12 of 119) than the SCS group (29 of 97, OR 0.263 , 95\% CI 0.126-0.550; $P<0.001$ ).

No difference in bile duct injury, mean intensive care unit or hospital stay (days), graft survival (at 1 year) or survival of the patient (at 1 year) was observed between the two groups. In addition, the proportion of patients with adverse events was comparable between the two groups.

"Transplanted livers were shown to function better if they had been preserved using a novel technology, NMP. The benefit was most pronounced in the most marginal donor livers, particularly those originating from DCD donors," notes Nasralla. "NMP was also associated with increased use of available donor organs compared to SCS - the number of livers retrieved from organ donors but then declined as unsuitable to transplant after preservation, was halved," he adds.

The researchers are now planning to extend the development of NMP for use with other transplant organs, in particular the kidney, and further work to push NMP towards the clinic. Author Constantin Coussios is hopeful. "The greatest risk faced by any patient on a waiting list (anywhere in the world) is that of dying before receiving a transplant. We believe that the widespread application of NMP will maximise the utilization of donor organs".

Katrina Ray

ORIGINAL ARTICLE Nasralla, D. et al. A randomized trial of normothermic preservation in liver transplantation. Nature https://doi.org/ 10.1038/s41586-018-0047-9 (2018) 